\title{
Cooperative Control of Multiple Electronic Combat Air Vehicles for Electronic Attack
}

\author{
Il-hyoung Lee ${ }^{1}$ and Hyochoong Bang ${ }^{2}$ \\ ${ }^{1}$ Division of Aerospace Engineering, Korea Advanced Institute of Science and Technology, Daejeon, Korea \\ (Tel : +82-42-869-5758; E-mail: ihlee@ascl.kaist.ac.kr) \\ ${ }^{2}$ Division of Aerospace Engineering, Korea Advanced Institute of Science and Technology, Daejeon, Korea \\ (Tel : +82-42-869-3722; E-mail: hcbang@ascl.kaist.ac.kr)
}

\begin{abstract}
This paper presents a cooperative control scheme for electronic attack of multiple Electronic Combat Air Vehicles (ECAVs). The stealthy ECAVs equipped with ECM can deceive the radar by using range delay deception technique. This makes the radar to detect a fake target called Phantom beyond the ECAV location. The important feature of generating phantom track is kinematic and dynamic constraints. These constraints restrict the freedom of Phantom tracks. This paper presents three-dimensional mathematical relationships between the motion of the ECAV and the motion of the phantom. Based on the mathematical model, the trajectory generation problem is formulated as an optimal control problem. The control input parameterization converts the optimal control problem into the parameter optimization problem with inequality constraints. This problem is solved by using the sequential quadratic programming method. The numerical result for the optimal phantom trajectory generation is presented.
\end{abstract}

Keywords: Optimal trajectory generation, ECAV, Phantom, three-dimensional

\section{INTRODUCTION}

Electronic warfare (EW) is the use of the electromagnetic spectrum to effectively deny the use of this medium by an adversary, while optimizing its use by friendly forces. Electronic warfare has three main components: electronic support (ES), electronic attack (EA), and electronic protection (EP). ES is the passive use of the electromagnetic spectrum to gain information about enemies on the battlefield in order to find, identify, locate and intercept potential threats or targets. EA is the active or passive use of the electromagnetic spectrum to deny its use by an adversary. EP includes all activities related to making enemy EA activities less successful by means of protecting friendly personnel, facilities, equipment or objectives. Nowadays Unmanned aerial Vehicles (UAV) such as Global hawk, Predator are used in real battle field and their capabilities are proved. And UCAV Unmanned Combat Aerial Vehicle (UCAV) fielding is attainable in the near future. Cost effective small Electronic Combat Air Vehicles (ECAV) with the intelligent cooperative control technique would play a very significant role in EA. The stealthy ECAVs equipped with ECM (Electronic Counter measures) can deceive the radar by using range delay deception technique. This make the radar to detect a fake target called Phantom beyond the ECAV location. The important feature of generating phantom track is kinematic and dynamic constraints. These constraints restrict the freedom of Phantom tracks. The problem is formulated in three dimensions. The phantom has three degree of freedom while each ECAV has a one degree of freedom. The reduction of the degree of freedom for the ECAV is due to the constraint that ECAV and the phantom should locate on the same line of sight from the radar to phantom. In this paper optimal cooperative control problem to deceive radar networks using multiple ECAVs is formulated to and some approaches to solving the technical problems are described.

\section{DECEPTION PROBLEM}

Assuming that an EACV is not detected using the stealthy technology, an ECAV can generate a phantom by intercepting and delaying the return of the radar's transmitted pulse. So the deceived radar sees a phantom beyond the ECAV. ECAV can only fake distance, the phantom is on the line of sight from the radar to the ECAV. Fig. 1 shows an example of a phantom track. Two EACs could cooperatively generate a single coherent phantom track. In this figure two radars share the information of the targets to get the fine information of them. Unless all radars see the same phantom track, the track is dismissed as spurious [1].

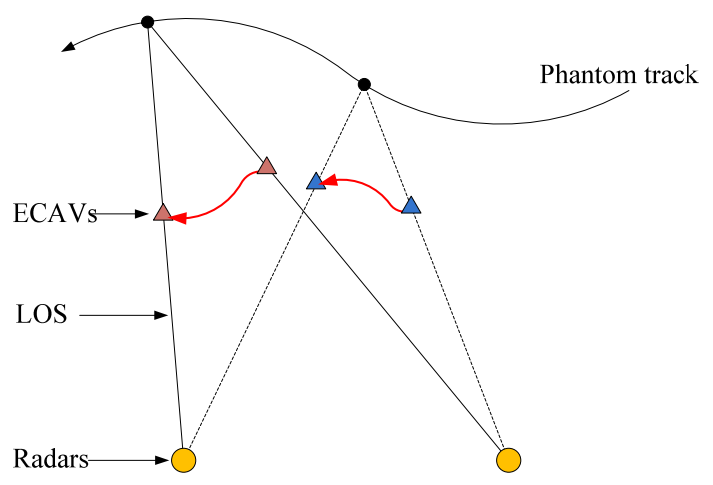

Fig. 1 Phantom track generation

\section{ECAV AND PHANTOM DYNAMICS}

In previous studies on this problem two-dimensional ECAV and phantom dynamics are derived in [1], [2], [4], [5] and three-dimensional phantom trajectory generation is studied in [3]. We expand the previous dynamics equations to three-dimensional equation of the motion in polar coordinate. The definitions of the main ECAV and phantom variables and their relations are shown in 
Fig. 2. Here $r, R$ are the radial distance from radar to ECAV and the radial distance from radar to phantom target. $x, y$ and $z$ denote the position coordinates in Cartesian coordinate. $\alpha$ and $\beta$ are the heading angle and flight path angle of the velocity vector. $\theta$ and $\varphi$ are bearing angle and azimuth angle from radar to ECAV and phantom target. Subscripts E and P denote ECAV and Phantom respectively.

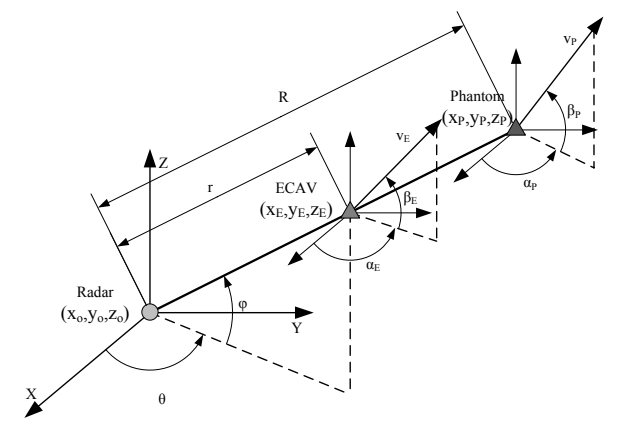

Fig. 2 ECAV and Phantom track variables and relations

For ease of comparison non-dimensional variables are used, with the $v_{m}=1$ and $R_{0}=1[1]$. Here $v_{m}$ is the nominal speed of ECAV and $R_{0}$ is the initial radial distance from radar to phantom target.

$$
r \rightarrow \frac{r}{R_{0}} \quad R \rightarrow \frac{R}{R_{0}} \quad t=\frac{v_{m}}{R_{0}} t \quad v_{P} \rightarrow \frac{v_{P}}{v_{m}}
$$

The basic equations of motion for the Phantom target in Cartesian coordinates are as follows.

$$
\begin{aligned}
& \dot{x}_{P}=v_{P} \cos \beta_{P} \cos \alpha_{P} \\
& \dot{y}_{P}=v_{P} \cos \beta_{P} \sin \alpha_{P} \\
& \dot{z}_{P}=v_{P} \sin \beta_{P} \\
& \dot{v}_{P}=u_{P 1} \\
& \dot{\alpha}_{P}=u_{P 2} \\
& \dot{\beta}_{P}=u_{P 3}
\end{aligned}
$$

where control inputs $u_{p 1}, u_{p 2}$ and $u_{p 3}$ are linear acceleration, heading rate and flight path angle rate, respectively. The equations of motion for the Phantom target using polar coordinates are as follows.

$$
\begin{aligned}
\dot{R}= & v_{P}\left(\cos \phi \cos \theta \cos \beta_{P} \cos \alpha_{P}\right. \\
& \left.+\cos \phi \sin \theta \cos \beta_{P} \sin \alpha_{P}+\sin \phi \sin \beta_{P}\right) \\
\dot{\theta}= & \frac{v_{P}}{R \cos \phi}\left(-\sin \theta \cos \beta_{P} \cos \alpha_{P}\right. \\
& \left.+\cos \theta \cos \beta_{P} \sin \alpha_{P}\right)
\end{aligned}
$$

$$
\begin{aligned}
\dot{\phi}= & \frac{v_{P}}{R}\left(-\sin \phi \cos \theta \cos \beta_{P} \cos \alpha_{P}\right. \\
& \left.-\sin \phi \sin \theta \cos \beta_{P} \sin \alpha_{P}+\cos \phi \sin \beta_{P}\right)
\end{aligned}
$$

From Eqs. (7) (8) equations for interfacing with the ECAV system are obtained.

$$
\begin{aligned}
\dot{\theta} & =\frac{\left(x_{P}-x_{o}\right) \dot{y}_{T}-\left(y_{P}-y_{o}\right) \dot{x}_{T}}{R_{x y}{ }^{2}} \\
\dot{\phi} & =\frac{\sqrt{\left(x_{P}-x_{o}\right)^{2}+\left(y_{P}-y_{o}\right)^{2}} \dot{z}}{R^{2}} \\
& -\frac{\left(z_{P}-z_{o}\right)}{R^{2}}\left(\frac{\left(x_{P}-x_{o}\right) \dot{x}}{R_{x y}}+\frac{\left(y_{P}-y_{o}\right) \dot{y}}{R_{x y}}\right) \\
\phi & =\sin ^{-1}\left(\frac{z_{P}-z_{o}}{R}\right) \\
\theta & =\tan ^{-1}\left(\frac{y_{P}-y_{o}}{x_{P}-x_{o}}\right)
\end{aligned}
$$

where $R_{x y}$ is $\sqrt{\left(x_{P}-x_{o}\right)^{2}+\left(y_{P}-y_{o}\right)^{2}}$.

The basic equations of motion for the ECAV in Cartesian coordinates are as follows. The equations are similar to the phantom dynamics.

$$
\begin{aligned}
\dot{r}= & v_{E}\left(\cos \phi \cos \theta \cos \beta_{E} \cos \alpha_{E}\right. \\
& \left.+\cos \phi \sin \theta \cos \beta_{E} \sin \alpha_{E}+\sin \phi \sin \beta_{E}\right) \\
\dot{\theta}= & \frac{v_{E}}{R \cos \phi}\left(-\sin \theta \cos \beta_{E} \cos \alpha_{E}\right. \\
& \left.+\cos \theta \cos \beta_{E} \sin \alpha_{E}\right) \\
\dot{\phi}= & \frac{v_{E}}{R}\left(-\sin \phi \cos \theta \cos \beta_{E} \cos \alpha_{E}\right. \\
& \left.-\sin \phi \sin \theta \cos \beta_{E} \sin \alpha_{E}+\cos \phi \sin \beta_{E}\right)
\end{aligned}
$$

For the geometrical constraint that ECAV lie on the line of sight from the radar to the phantom. The ECAV has the single degree of freedom. One dynamic equation and one control input is enough for the ECAV equation of motion. From Eqs (14)-(16) the basic equation of motion can be derived as follows.

$$
\begin{aligned}
& \tan \beta_{E}=\frac{\cos \phi \sin \phi \cos \left(\alpha_{E}-\theta\right) \dot{\theta}+\sin \left(\alpha_{E}-\theta\right) \dot{\phi}}{\cos ^{2} \phi \dot{\theta}} \\
& \dot{r}=r \dot{\theta}\left(\cos ^{2} \phi \cot \left(\alpha_{E}-\theta\right)+\tan \beta_{E} \frac{\cos \phi \sin \phi}{\sin \left(\alpha_{E}-\theta\right)}\right. \\
& \dot{\alpha}_{E}=u_{E 2}
\end{aligned}
$$

Where control input $u_{E 2}$ is ECAV heading rate. The constrained parameters such as $\theta, \dot{\theta}, \phi$ and $\dot{\phi}$ are obtained from the interfacing equations of the phantom track Eqs. (10) (13). ECAV velocity is obtained as follow.

$$
v_{E}^{2}=\dot{r}^{2}+(r \cos \phi \dot{\theta})^{2}+(r \dot{\phi})^{2}
$$




\section{OPTIMAL TRAJECTORY GENERATION}

In descrete time, optimal trajectory generation problem is

$$
\min _{u_{k}} J=\Phi\left(x_{N}, N\right)+h \sum_{k=0}^{N-1} L\left(x_{k}, u_{k}, k\right)
$$

Subject to

$$
\begin{aligned}
& x_{k+1}=f\left(x_{k}, u_{k}, k\right) \\
& C_{e q}\left(x_{k}, u_{k}, k\right)=0 \\
& C\left(x_{k}, u_{k}, k\right) \leq 0
\end{aligned}
$$

Where $N=t_{f} h, \mathrm{~h}$ is time step for descritization. The parameter optimization technique is used to solve this problem. Control inputs are parameterized and convert the optimal control problem into finite-dimensional, constrained parameter optimization problem to find the control input history $u_{k}$ minimize the cost function as follows. The control input can be parameterized by $X=\left[u_{0} u_{1} \cdots u_{N-1}\right]^{T}$ as shown in Fig. $3 . \mathrm{X}$ is the parameter vector for the optimization problem.

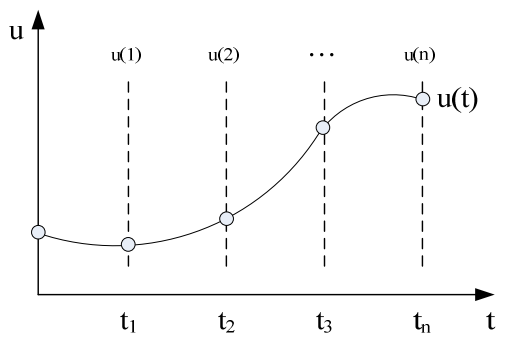

Fig. 3 Control input parameterization

The converted parameter optimization problem is

$$
\begin{aligned}
& \min _{X} f_{J}(X) \\
& \text { Subject to } \\
& g(X) \leq 0 \\
& g_{e q}(X)=0
\end{aligned}
$$

$f_{J}, g$ and $g_{e q}$ are cost function, nonlinear inequality constraints and nonlinear equality constraints, respectively.[2]

In this paper cost function for the optimal control problems is set

$$
J=\sum_{0}^{N-1}\left(c_{1} r_{k}^{2}+c_{2} u_{E 2}^{2}\right)
$$

The cost function minimizes the distance between ECAV and radar and the curvature of the ECAV trajectory. The ECAV's linear speed and heading rate are limited due to the physical dynamic limitation.

$$
\begin{aligned}
& v_{\text {min }} \leq v_{E} \leq v_{\text {max }} \\
& u_{E 2 \min } \leq u_{E 2} \leq u_{E 2 \max }
\end{aligned}
$$

\section{RESULTS}

Simulation results of the optimal control algorithm for the case of three ECAVs engaging 3 radars are performed. The total cost of this problem is summation of the each cost of the ECAV. The solutions are obtained using $\mathrm{C}$ code for Feasible Sequential Quadratic Programming(CFSQP).

The three dimensional simulation results are shown in Fig 4. The trajectory of each ECAV is lied on the line of sight from the radar to phantom.

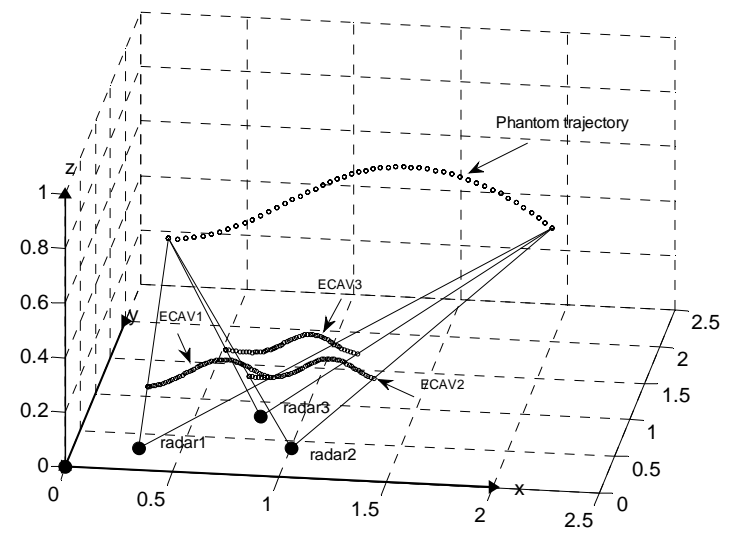

Fig. 4 Simulation results of trajectories for three ECAVs engaging three radars generating a three dimensional coherent phantom track

Fig 5 and Fig 6 illustrate the projection on $x y$-plane and $x z$-plane of the phantom trajectory of a $3 \mathrm{D}$ path.

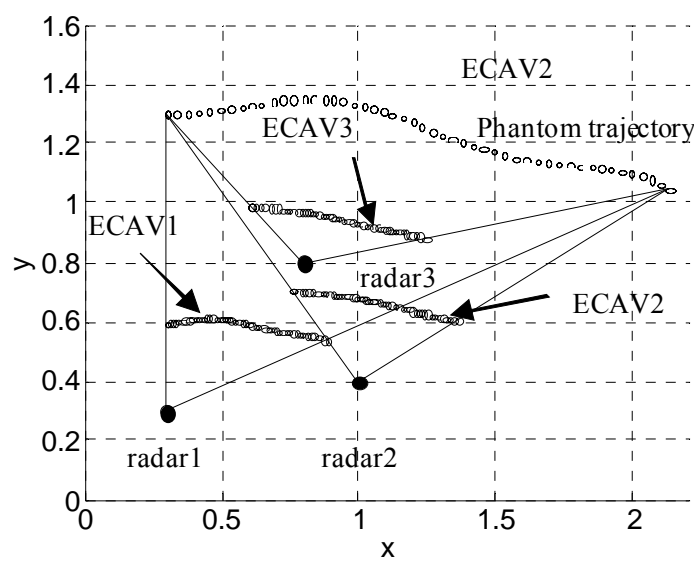

Fig. 5 Projection on $x y$-plane of the phantom trajectory of a $3 \mathrm{D}$ path

Constrained speeds of the ECAVs are shown in Fig 7. Dash-lines are the maximum and minimum bounds of the each ECAV. The speeds of the all ECAVs are bounded to the given speed constraint. Fig. 7 gives the simulation results for the control inputs(heading rate). When the speed of the ECAV reaches the speed limit bound, the heading rate curve become steep. Constraint violation is avoided due to fast change of the heading rate. 


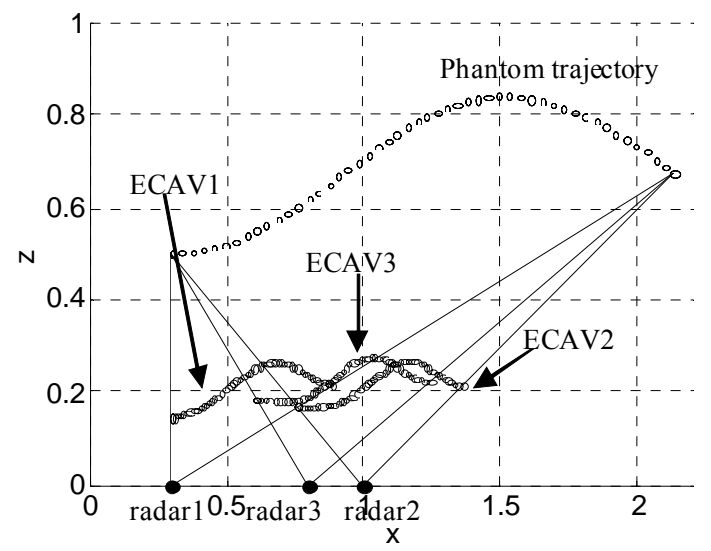

Fig. 6 Projection on $x z$-plane of the phantom trajectory of a $3 \mathrm{D}$ path
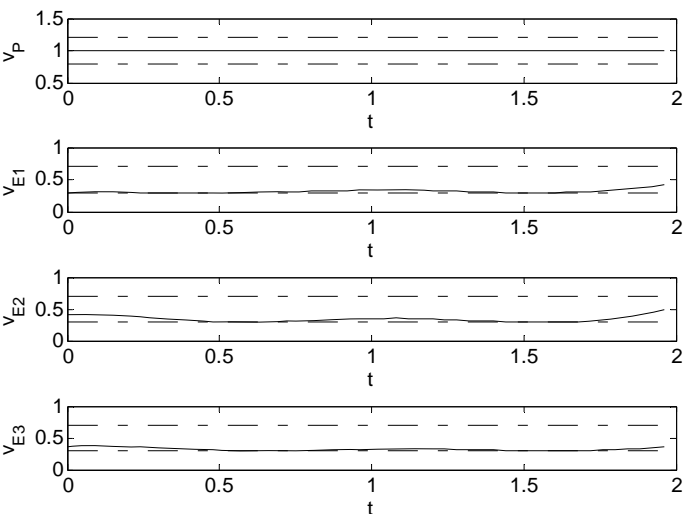

Fig. 7 Constrained speeds of the ECAVs and phantom target
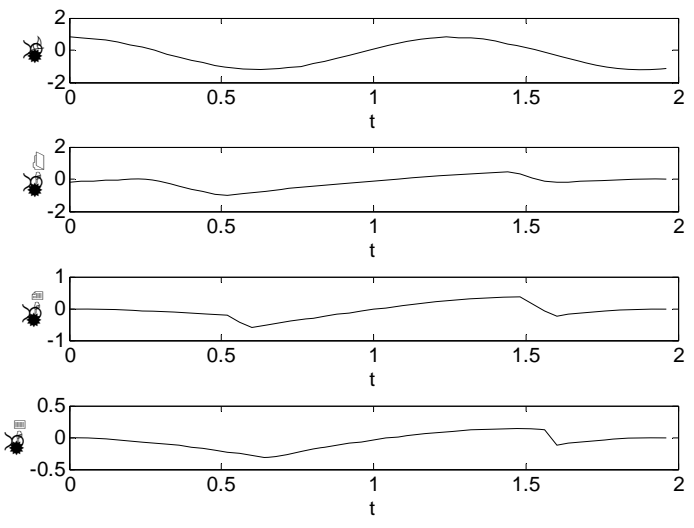

Fig. 8 Heading-rate control inputs of the ECAVs and phantom target

\section{CONCLUSION}

In this paper the application of cooperative control of multiple ECAV in EA has been considered. Especially the mission of deceiving a network of radar using phantom trajectory generation is investigated. Three-dimensional mathematical relationships between the motion of the ECAV and the motion of the phantom is derived and based on this mathematical model, the trajectory generation problem is formulated as an optimal control problem. The control input parameterization converts the optimal control problem into the parameter optimization problem with inequality constraints. This problem is solved by using the CFSQP. The numerical result for three dimensional optimal phantom trajectory generation is presented.

\section{REFERENCES}

[1] K. B. Purvis, P. R. Chandler, M. Pacher, "Feasible Flight Paths for Cooperative Generation of a Phantom Radar Track," Journal of Guidance, Control, and Dynamics, Vol. 29, No. 3, 2006, pp. 653-661.

[2] Keith B. Purvis, Karl J. Astrom, Mustafa Khammash, "Online Control Strategies for Highly Coupled Cooperative UAVs", Proceedings for the American Control Conference, pp. 653-661,New York City, USA, 2007.

[3] D. H. A. Maithripala, S. Jayasuriya, "Phantom Track Generation in 3D through Cooperative Control of Multiple ECAVs Based on Geometry," First International Conference on Industrial and Information Systems, ICIIS 2006, Sri Lanka, 2006.

[4] M. Pachter, P. R. Chandler, R. A. Larson, K. B. Purvis, "Concepts for Generating Coherent Radar Phantom Tracks Using Cooperating Vehicles," AIAA Guidance, Navigation, and Control Conference and Exhibit, 2004.

[5] D. H. A Maithripala, S. Jayasuriya, M. J. Mears, "Phantom Track Generation Through Cooperative Control of Multiple ECAVs Based on Feasibility Analysis," Journal of Dynamic Systems, Measurement, and Control, Vol. 129, 2007, pp. 709-715.

[6] M. J. Mears, M. R. Akella, "Deception of RADAR Systems using Cooperatively controlled Unmanned Air Vehicles," Networking, Sensing and Control, Proceedings, 2005.

[7] D. H. A. Maithripala and S. Jayasuriya, "Radar Deception through Phantom Tack Generation," American Control Conference, Portland, OR, 2005.

[8] J. Arthur E, Bryson and Y.C. HO, Applied Optimal Control, John Wiley \& Sons, New York, 1975.

[9] F. L. Lewis, Optimal Control, John Wiley \& Sons, 1986.

[10] C. Lawrence, J. Zhou, \& J. Tits. (1997). User's Guide for CFSQP Version 2.5. University of Maryland, Electrical Engineering Dept \& Institute for Systems Research. 